\title{
Temperature dependence of the nuclear symmetry energy
}

\author{
D. J. Dean, S. E. Koonin, K. Langanke, and P. B. Radha \\ W. K. Kellogg Radiation Laboratory, 106-38, California Institute of Technology \\ Pasadena, California 91125 USA
}

(March 6, 2018)

\begin{abstract}
We have studied the properties of $A=54$ and $A=64$ isobars at temperatures $T \leq 2 \mathrm{MeV}$ via Monte Carlo shell model calculations with two different residual interactions. In accord with empirical indications, we find that the symmetry energy coefficient, $b_{\text {sym }}$, is independent of temperature to within $0.6 \mathrm{MeV}$ for $T \leq 1 \mathrm{MeV}$. This is in contrast to a recent suggestion of a $2.5 \mathrm{MeV}$ increase of $b_{\text {sym }}$ for this temperature, which would have significantly altered the supernova explosion scenario.
\end{abstract}


There appears to be a general consensus on the basic scenario for presupernova collapse. An iron core is produced in the center of a massive star in the final stage of hydrostatic nucleosynthesis. When the iron core exceeds the Chandrasekhar mass limit, it can no longer be supported by electron degeneracy pressure and so begins to collapse. Subsequent photodisintegration of nuclei and electron capture on nuclei (later on free protons) reduce the pressure and energy of the core and accelerate its collapse, in this way raising the central density and temperature. After neutrino trapping, the inner part of the core (mass densities $\rho \gtrsim 10^{12} \mathrm{~g} / \mathrm{cm}^{3}$ ) collapses as a homologous unit whose size is given by the appropriate Chandrasekhar mass limit, $M_{\mathrm{Ch}} \approx 5.76\left(Y_{e}^{\text {trap }}\right)^{2} M_{\odot}$, where $Y_{e}^{\text {trap }}$ is the electron-to-nucleon ratio of the trapped material. The collapse halts when the homologous core exceeds nuclear matter density $\left(\rho \gtrsim 10^{14} \mathrm{~g} / \mathrm{cm}^{3}\right)$. A shock wave, formed at the inner core's surface, then travels outward and eventually explodes the star.

Despite the appeal of this scenario, the actual mechanism of a supernova explosion is still controversial. In the direct mechanism the shock wave is strong enough not only to stop the collapse, but also to explode the outer shells. In the delayed mechanism the shock energy is first stored in the core mantle (the layer of infalling material in the iron core outside the homologous region), but it is recovered shortly thereafter by the outstreaming neutrinos. A detailed description of the physics of a supernova can be found in Ref. [1].

The size of the core mantle is a major determinant of the supernova mechanism. It has been argued recently that the mantle size might be significantly smaller than generally calculated in supernova models because of an overlooked temperature dependence of the electron capture process [2]. The detailed reasoning is as follows [3] : When the nucleus is described by a static mean field model, dynamical effects associated with collective surface vibrations are conventionally embodied in an effective nucleon mass, $m^{*}$ (as are spatial nonlocalities in the mean field). Donati et al. [3] studied the coupling of the mean field singleparticle levels to the collective surface vibrations within the quasiparticle random phase approximation and found that $m^{*}$ decreases with increasing temperature for $T \leq 2 \mathrm{MeV}$, the temperatures relevant for the presupernova collapse. They attributed this behavior to a reduction of collectivity at low excitation energies. In the Fermi gas model, this decrease of $m^{*}$ induces an increase in the symmetry energy contribution to the nuclear binding energy

$$
E_{\mathrm{sym}}(T)=b_{\mathrm{sym}}(T) \frac{(N-Z)^{2}}{A},
$$

where $N, Z$, and $A$ are the neutron, proton, and mass numbers of the nucleus, respectively. Quantitatively, Donati et al. estimate that $b_{\mathrm{sym}}(T)$ increases by about $2.5 \mathrm{MeV}$ as $T$ increases from 0 to $1 \mathrm{MeV}\left(b_{\text {sym }}(0) \approx 28 \mathrm{MeV}\right.$ 四 $)$.

Importantly, a larger value of $E_{\mathrm{sym}}(T)$ would hinder electron capture in the presupernova environment, and thus increase $Y_{e}^{\text {trap }}$. This, in turn, would reduce the size of the core mantle so that the shock wave would need significantly less energy to stop the collapse and explode the star. In the supernova scenario outlined by Donati et al., the temperature dependence of $b_{\text {sym }}$ increases the shock energy by about 0.5 foe, roughly half of the observed explosion energy of SN87a [3].

A more definitive test of the proposed temperature dependence of the nuclear symmetry energy (and hence of the presupernova electron capture process) necessarily involves a proper account of those nuclear degrees of freedom beyond the mean field (e.g., pairing correlations) 
that are relevant for nuclei under presupernova conditions (e.g., $f p$-shell nuclei at $T \leq$ $2 \mathrm{MeV}$ ). The model of choice for this task is the interacting shell model, which provides a complete nuclear spectrum and so allows a statistical description using a canonical ensemble; both quantal and thermal fluctuations (within the model space chosen) are fully included.

The recent development of the Monte Carlo shell model [5] has made feasible complete $(0 \hbar \omega)$ calculations of $f p$-shell nuclei at finite temperature; a pilot study of the thermal properties of the nucleus ${ }^{54} \mathrm{Fe}$ has been presented in Ref. [6]. To explore the temperature dependence of the symmetry energy, we have used this method to study the thermal properties of two isobar chains $(A=54$ and 64$)$ in the mass region important for the presupernova collapse; this includes two of the three nuclei studied in Ref. [3] ( ${ }^{64} \mathrm{Zn}$ and ${ }^{64} \mathrm{Ni}$ ). Technical considerations restrict our Monte Carlo shell model calculations to the $N=Z$ and even-even members of the chains. The details of our calculation parallel those of the ${ }^{54} \mathrm{Fe}$ study in Ref. [6] and the Monte Carlo shell model is described in detail in Refs. [5.:7]. For the $A=54$ isobar chain $\left({ }_{27}^{54} \mathrm{Co},{ }_{28}^{54} \mathrm{Fe},{ }_{30}^{54} \mathrm{Cr}\right.$, and $\left.{ }_{32}^{54} \mathrm{Ti}\right)$ we have adopted the Brown-Richter interaction [8]. For the $A=64$ isobars $\left({ }_{32}^{64} \mathrm{Ge},{ }_{34}^{64} \mathrm{Zn},{ }_{36}^{64} \mathrm{Ni}\right.$, and $\left.{ }_{38}^{64} \mathrm{Fe}\right)$, for which the Brown-Richter force is expected to be unreliable [9] (it was constructed from properties of nuclei at the beginning of the $f p$-shell), we have used the KB3 residual interaction [10]. Further, to explore the sensitivity of our results to the two-body interaction, we have also studied the $A=54$ chain with the KB3 interaction. All of our calculations omit the Coulomb interaction and assume complete isospin symmetry.
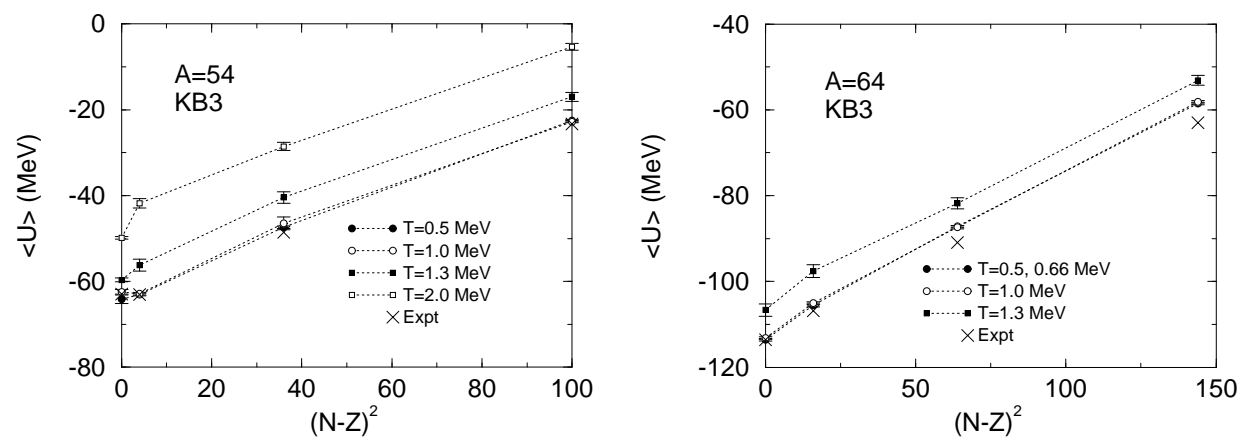

Figure 1. Calculated internal energies, $U$, for the $A=54$ and $A=64$ isobar chains at various temperatures using the KB3 interaction. Results for $A=54$ with the Brown-Richter interaction are virtually identical. The energies are given relative to the doubly-magic nucleus ${ }^{40} \mathrm{Ca}$. The experimental energies have been corrected for the Coulomb repulsion as described in the text and, in the case of $A=64$, have been increased by $11 \mathrm{MeV}$ to account for the systematic underbinding of the Hamiltonian for these isobars.

In Fig. 1 we display our results for $U$, the internal energy (expectation value of the Hamiltonian in the canonical ensemble) for the $A=54$ isobars and $A=64$ isobars as a function of $(N-Z)^{2}$ at temperatures relevant for the presupernova. For even-even nuclei our calculation at $T=0.5 \mathrm{MeV}$ has almost cooled to the ground state, as the first excited states in these nuclei have excitation energies $E_{x} \gtrsim 1 \mathrm{MeV}$. For the $N=Z$ nucleus ${ }^{54} \mathrm{Co}$, the $T=0.5 \mathrm{MeV}$ calculation represents a mixture of the ground state and the first excited state at $200 \mathrm{keV}$. If we correct the experimental binding energies by the semi-empirical Coulomb 
energy, $E_{c}=0.717 Z^{2} / A^{1 / 3}\left(1-1.69 / A^{2 / 3}\right) \mathrm{MeV}$ [11], we find that our calculation with either interaction accurately reproduces the $A=54$ binding energies. For the $A=64$ nuclei, the low-temperature calculations are systematically overbound by some $11 \mathrm{MeV}$, although the variation along the chain is well reproduced. A better description of the nuclei ${ }^{64} \mathrm{Ni}$ and ${ }^{64} \mathrm{Fe}$ (with 16 and 18 valence neutrons in the $f p$-shell) would plausibly require inclusion of the $g_{9 / 2}$ orbital. We observe that, at a given temperature, $U$ is linearly proportional to $(N-Z)^{2}$ for the even-even nuclei of an isobar chain. This behavior is, of course, expected from semiempirical parametrizations of the binding energy (e.g., the Bethe-Weizsäcker formula) and almost exclusively reflects the symmetry energy contribution to the binding energies (1).

For $A=54$, our calculation yields $b_{\text {sym }}(0)=21 \pm 1.0 \mathrm{MeV}$ and $22 \pm 0.75 \mathrm{MeV}$ for the KB3 and Brown-Richter interactions, respectively. These values are in very good agreement with experiment, $b_{\text {sym }}=21 \mathrm{MeV}$. For $A=64$, we find $b_{\text {sym }}=23 \pm 0.7 \mathrm{MeV}$, slightly larger than the experimental value of $21.2 \mathrm{MeV}$. We note that all of these values are significantly smaller than the symmetry energy coefficient adopted in presupernova studies $\left(b_{\text {sym }}=30-32 \mathrm{MeV}\right.$ [2,12, 12 .

Our calculation does not confirm the temperature dependence of the symmetry energy proposed in Ref. [3]. As is obvious from Fig. 1, the slope of the linear variation of $U$ with $(N-Z)^{2}$ for the even-even nuclei is independent of temperature, indicating that $b_{\text {sym }}$ does not change. To be more quantitative, an increase of $b_{\text {sym }}$ by $2.5 \mathrm{MeV}$ between $T=0$ and $1 \mathrm{MeV}$, as proposed in Ref. [3], corresponds to an increase in the energy splitting between ${ }^{54} \mathrm{Fe}$ and ${ }^{54} \mathrm{Ti}$ of $\Delta=4.4 \mathrm{MeV}$ (using the experimental value for $b_{\text {sym }}$ ), while our calculation yields $\Delta=0.4 \pm 0.9 \mathrm{MeV}$, implying that $b_{\text {sym }}$ changes by less than $0.7 \mathrm{MeV}$. In fact, our calculation shows that all properties of the even-even nuclei studied here are essentially constant for $T \leq 1 \mathrm{MeV}$. These include the quadrupole strength, whose variation with temperature has been cited as the source for the proposed temperature-dependence of the symmetry energy in Ref. [3].

The low-temperature constancy of the properties of even-even nuclei is expected in view of their sparse spectrum of low-lying excited states and relatively high excitation energies of their first excited states. To confirm this, we have calculated the nuclear thermodynamics from the low-lying experimental levels of the nuclei in the two isobar chains; enough of these levels are known to give reliable results up to $T=0.7 \mathrm{MeV}$. The partition function is given by

$$
Z(T)=\sum_{J}(2 J+1) e^{-E_{J} / T}
$$

where the free energy is given by $F(T)=-T \ln Z(T)$, and the internal energy is $U=$ $-T^{2} \partial(F / T) / \partial T$. We then calculated the difference in internal energies between isobars $a$ and $b$ as a function of the temperature, and expressed that in terms of $b_{\text {sym }}$ through Eq. (1).

For the $A=54$ chain, we used all known levels in ${ }^{54} \mathrm{Fe}$ and ${ }^{54} \mathrm{Cr}$ up to $10 \mathrm{MeV}$ of excitation energy; levels with unknown spin were assigned a $J$-value randomly chosen from the standard empirical spin distribution function [13]. Results for $U$ are shown in the lefthand panel of Fig. 2, expressed in terms of $\Delta b_{\text {sym }}$, the change in $b_{\text {sym }}$ relative to its value at $T=0$. There is structure in the curve due to the peculiarities of the ${ }^{54} \mathrm{Fe}$ and ${ }^{54} \mathrm{Cr}$ spectra, although it is smaller than the precision of our Monte Carlo calculations. We find that $\left|b_{\text {sym }}\right|$ changes by $0.3 \mathrm{MeV}$ at most as $T$ varies from 0 to $0.7 \mathrm{MeV}$. A similar calculation in the 
$A=64$ chain using ${ }^{64} \mathrm{Zn}$ and ${ }^{64} \mathrm{Ni}$ gives a change in $\left|b_{\text {sym }}\right|$ of $0.6 \mathrm{MeV}$ as $T$ varies from 0 to $0.6 \mathrm{MeV}$. In contrast, Donati et al. claim that $b_{\text {sym }}$ increases by $1.22(1.53) \mathrm{MeV}$ for ${ }^{64} \mathrm{Ni}$ $\left({ }^{64} \mathrm{Zn}\right)$ as $T$ varies from 0 to $0.7 \mathrm{MeV}$. Note that although we have defined the symmetry energy coefficient in terms of $U$, rather than $F$, the right-hand panel of Fig. 2 shows that the symmetry coefficient of $F$ never varies by more than $0.5 \mathrm{MeV}$ for $T<0.7 \mathrm{MeV}$. It is also interesting to note that, depending on the low-lying spectra, $b_{\text {sym }}$ may either increase or decrease as $T$ increases.
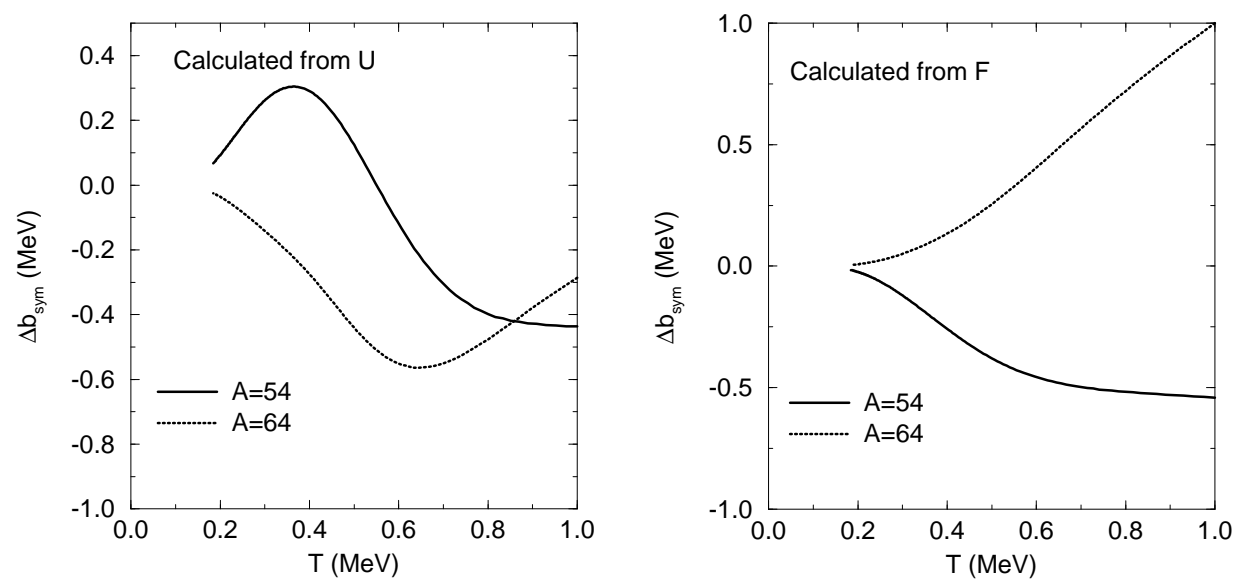

Figure 2. Temperature dependence of the symmetry energy coefficients for the internal energy, $U$, and free energy, $F$, calculated from the empirical spectra as described in the text. ${ }^{54} \mathrm{Fe}$ and ${ }^{54} \mathrm{Cr}$ were compared to calculate the $A=54$ case, while ${ }^{64} \mathrm{Zn}$ and ${ }^{64} \mathrm{Ni}$ were used for $A=64$. The limited knowledge of levels at high excitation energy makes these cases unreliable for $T \gtrsim 0.7 \mathrm{MeV}$.

In contrast to even-even nuclei, odd-odd nuclei (with $N$ not equal to $Z$ ) have a rather rich spectrum at low excitation energies. Thus, $U$ is expected to increase more strongly for these nuclei at temperatures $T<1 \mathrm{MeV}$. From a simple Fermi gas picture one expects an increase of $a T^{2} \approx 7 \mathrm{MeV}$, with the empirical level density parameter $a=A / 8 \mathrm{MeV}^{-1}$ [13. The increase of $U$ with temperature for odd-odd nuclei changes the kinematics of electron capture, making it easier for odd-odd nuclei to capture electrons.

As is demonstrated in detail in Ref. [6] for the case of ${ }^{54} \mathrm{Fe}$, our Monte Carlo shell model calculations predict the disappearance of pairing correlations between like nucleons at $T \approx 1.3 \mathrm{MeV}$, while $n p$-correlations persist to higher temperatures. We observe the same behavior for the nuclei studied here. At $T \approx 1.3 \mathrm{MeV}$ we find a significant change in the thermal properties of the nuclei. For example, the moment of inertia and the (orbital) $M 1$ strength increase drastically. This change is also apparent from the increase of $U$ with temperature, as shown in Fig. 1. Monitored by the number of BCS-like pairs in the nuclei,

\footnotetext{
${ }^{1}$ We have calculated the change of the symmetry energy coefficient predicted by Donati et al. using the exponential fit formula given in Table I of Ref. [3]. We note, however, that this formula significantly underestimates the change in $b_{\text {sym }}$ at $T=1 \mathrm{MeV}$ compared to the QRPA results given in the same table.
} 
we observe that the change is related to the disappearance of the $p p$ - and $n n$-correlations, which, in turn, dominate the nuclear properties at low temperatures. The vanishing of the pairing energy in even-even nuclei at $T \geq 1.3 \mathrm{MeV}$ has a significant effect on the kinematics of the electron capture on nuclei in the late stage of presupernova collapse.

Another novel feature is apparent in our calculations, although it has no direct relation to the presupernova situation. It is well known that odd-odd $N=Z$ nuclei are slightly less bound relative to the even-even nuclei in an isobar chain because of the lack of like-particle pairing of the last proton and neutron. However, there is apparently a strong correlation between these unpaired nucleons in $N=Z$ nuclei that energetically favors them over the other odd-odd nuclei in the chain. For both interactions, we observe in the $A=54$ isobar chain that ${ }^{54} \mathrm{Co}$ becomes energetically favored relative to the even-even nuclei, after the disappearance of the like-pair correlations in the latter. This observation is in agreement with the finding in [6] that isoscalar $n p$-correlations persist to higher temperatures than the like-pair correlations. Apparently these correlations still contribute to the internal energy of ${ }^{54} \mathrm{Co}$ at $T \geq 1.3 \mathrm{MeV}$.

In conclusion, Monte Carlo shell model calculations show clearly that pairing correlations play a decisive role in nuclear properties at low temperatures. For all $(N=Z$ and eveneven) $f p$-shell nuclei studied, we find that the nuclear properties are essentially constant for $T \leq 1 \mathrm{MeV}$, as expected from the spectrum of these nuclei. In particular, we find that the symmetry energy coefficient is constant to within some $0.6 \mathrm{MeV}$ for $T<1 \mathrm{MeV}$. Thus, our calculation does not confirm speculations based on a mean-field approximation that the symmetry energy increases by $2.5 \mathrm{MeV}$ in the temperature interval $T=0-1 \mathrm{MeV}$. Our calculations illustrate that nucleon-nucleon correlations beyond the mean-field level must be taken into account. A detailed account of this work will be given elsewhere.

This work was supported in part by the National Science Foundation, Grants No. PHY90-13248 and PHY91-15574. We thank the Concurrent Supercomputing Consortium for a grant of DELTA and PARAGON time. 


\section{REFERENCES}

[1] H. A. Bethe, Rev. Mod. Phys. 62 (1990) 801.

[2] G. E. Brown, in: Nuclei in the Universe, eds. M. W. Guidry and M. R. Strayer (Hilger, London, 1993).

[3] P. Donati, P. M. Pizzechero, P. F. Bortignon, and R. A. Broglia, Phys. Rev. Lett. 72 (1994) 2835.

[4] A. Bohr and B. R. Mottelson, Nuclear Structure (Benjamin, New York, 1964) Vols. I and II.

[5] G. H. Lang, C. W. Johnson, S. E. Koonin, and W. E. Ormand, Phys. Rev. C 48 (1993) 1518; N. Auerbach, G. F. Bertsch, B. A. Brown, and L. Zhao, Nucl. Phys. A 556 (1993) 190.

[6] D. J. Dean, S. E. Koonin, K. Langanke, and P. B. Radha, submitted to Phys. Rev. Lett.

[7] Y. Alhassid, D. J. Dean, S. E. Koonin, G. Lang, and W. E. Ormand, Phys. Rev. Lett. 72 (1994) 613.

[8] W. A. Richter, M. G. Vandermerwe, R. E. Julies, and B. A. Brown, Nucl. Phys. A523 (1991) 325.

[9] A. Poves, private communication.

[10] E. Caurier, A. P. Zuker, A. Poves, and G. Martinez-Pinedo, Phys. Rev. C... (1994) July

[11] W. D. Myers and W. J. Swiatecki, Nucl. Phys. 81 (1966) 1.

[12] G. Baym, H. A. Bethe, and C. Pethick, Nucl. Phys. A175 (1971) 225.

[13] J. J. Cowan, F.-K. Thielemann, and J. W. Truran, Phys. Rep. 208 (1991) 267. 